\title{
Niveles de consanguinidad en un rebaño lechero y su efecto en la producción y reproducción
}

\author{
Levels of inbreeding in a dairy herd and its effect on production and reproduction
}

Jorge P. Calderón Velásquez'; Gladys L. Garay Livia²

\begin{abstract}
Resumen
Se ha evaluado la población activa de vacas de la raza Brown Swiss de una explotación lechera intensiva en la costa central, con la finalidad de poder establecer el efecto de la consanguinidad o la depresión endogámica sobre las características productivas y reproductivas en ese rebaño. El tamaño de la población inicial fue de 136 animales hembras, generando una genealogía de 820 animales en 15 generaciones. La determinación de los valores de consanguinidad de los animales activos, como los genealógicos, fueron estimados mediante el programa ENDOG. De la población de referencia, se ha determinado que el grado de consanguinidad media para esa población fue de $F_{x}=2,07 \%$, con un valor máximo de $F_{x}=12,14 \%$, existiendo 21 animales no consanguíneos y 115 animales consanguíneos. Cuando se analizó los valores de consanguinidad por generaciones, se encontró que en las dos últimas generaciones todos los animales fueron consanguíneos. Se han formado cinco grupos de acuerdo con el valor de la consanguinidad, siendo el primer grupo los animales no consanguíneos y el resto con un intervalo de $2 \%$ de consanguinidad. No se ha encontrado estadísticamente un efecto negativo de la consanguinidad en la edad y en el número de servicios con el primer parto; en cambio, cuando se analizó el efecto sobre las lactaciones y el número de servicios de acuerdo con el nivel de consanguinidad, se encontró que los animales no consanguíneos mostraron los mejores valores; este hallazgo resultó ser un efecto significativo. El intervalo entre partos, estadísticamente no significativo, mostró mayores valores en los grupos consanguíneos. En cuanto al efecto sobre la producción de leche, fue muy significativo, mostrando los grupos consanguíneos los mejores valores de producción (producción corregida a 305d, $2 \mathrm{X}$ y edad adulta).Como los intervalos entre partos se alargan, hay mayor número de servicios y los Días en Leche (DIM) se ven afectados, por tanto se deben promover sistemas de apareamiento con una menor consanguinidad, con la finalidad de lograr mejores valores o índices reproductivos y productivos.
\end{abstract}

Palabras clave: consanguinidad; caracteres productivos; reproductivos en vacunos.

\begin{abstract}
We evaluated the active population of Brown Swiss cows in an intensive dairy farming located in the central coast, with the main objective of establishing the inbreeding effect or endogamy depression on the productive and reproductive characteristics of this herd. The initial population size was 136 dairy cows, the complete pedigree has 820 animals, in 15 generations. The coefficient of inbreeding was calculated using ENDOG software. From the base population of dairy cattle, the average coefficient of inbreeding was $\mathrm{F}_{\mathrm{x}}=2,07 \%$, the maximum value showed was $\mathrm{F}_{\mathrm{x}}=12,14 \%$, existing 21 not inbreed cows and 115 inbreed cows. When the inbreeding values by generation were analyzed we found that in the last two generations, all cows were inbreed. According to the coefficient of inbreeding value, we formed five groups, the first group was not inbreed and the following groups had an inbreed interval of $2 \%$. Statistically we didn't find a negative effect of inbreeding on age and number of inseminations with first pregnancy, however when we analyzed the effect over lactations and the number of inseminations according to level of inbreeding, we found that not inbreed animals had the highest values, which resulted in a significant effect. The fresh interval, was not significant statistically, but showed higher values on the inbreed groups. When it comes to milk production the results were very significant, with the highest inbreed groups showing the best production values (305d, 2X, Maturity age). Because the fresh interval becomes longer, there are more inseminations and the Milk Days are affected, the breeder must use systems with minimal inbreeding, to obtain better values on reproductive and productive traits.
\end{abstract}

Keywords: inbreeding; productive; reproductive traits; dairy cattle.

1 Profesor Principal, Departamento Académico de Producción Animal, Facultad de Zootecnia. Universidad Nacional Agraria La Molina, Lima, Perú. Email: jcalderonv@lamolina.edu.pe

2 Profesor Auxiliar, Escuela Profesional Académica de Agronomía. Universidad Nacional Santiago Antúnez de Mayolo, Barranca, Perú. 


\section{Introducción}

La producción intensiva de vacunos lecheros lleva a la utilización de técnicas reproductivas como la inseminación artificial, en la que se puede realizar una selección de los reproductores que serán los responsables de la mejora genética del rebaño en la producción de leche y de caracteres funcionales de tipo.

En el ganado Brown Swiss se observa que la producción de reproductores machos está concentrada en pocas familias, lo cual hace un poco difícil tener apareamientos de animales que no tengan un grado de parentesco, ya que ello conllevará tener animales consanguíneos que pueden afectar la expresión de las características de importancia económica, cuyo efecto es medido mediante la depresión endogámica.

El objetivo general del presente estudio es cuantificar el nivel de consanguinidad en un establo lechero comercial de la raza Brown Swiss, en el que se utiliza inseminación artificial (IA) como técnica reproductiva, así como producción y trasplantes de embriones. Se analizará como objetivos específicos la depresión endogámica que puedan causar los distintos niveles de consanguinidad en la producción de leche y los caracteres reproductivos, sea en vacas que en vaquillas; asimismo, se tomará en cuenta el número de servicios para la preñez efectiva del primer parto y en los sucesivos, edad al primer parto, intervalo entre partos, días de lactación (DIM) y producción de leche.

\section{Materiales y métodos}

\section{Revisión de literatura}

\section{Efectos sobre la producción de leche y reproducción}

Según Neira (1985), este tipo de cruzamientos (consanguíneos) debe tratar de evitarse porque produce efectos negativos en la capacidad reproductiva o eficiencia fisiológica de los individuos. Este fenómeno se conoce como depresión endogámica o depresión debido a la consanguinidad. El cruzamiento consanguíneo intensivo ha sido asociado a disminuciones en la producción de leche y de grasa en los vacunos lecheros; así como también a incrementos en las tasas de mortalidad, al ser comparado con individuos no consanguíneos.

Algunas de las consecuencias de la endogamia son una disminución de los rendimientos productivos, de los índices reproductivos y la exteriorización de caracteres letales, como terneros con cabeza de bulldog que causan la muerte de los individuos o subletales como criptorquidia, hernias y defectos de pezuña, que disminuyen su vitalidad y vida productiva (Mujica, 1992).

Según Cassell (1999), la selección para una alta producción y un mejor tipo lechero ha reducido la diversidad genética. Hoy, un número limitado de toros tiene una alta influencia sobre la siguiente generación dentro de la raza, la razón de esto es que se usan solo los toros con mayor mérito, los cuales son pocos.

En un estudio, Wiggans et al. (1995), midieron el nivel de consanguinidad en las razas lecheras en los Estados Unidos de Norteamérica, en el que se puede observar que todas las razas muestran ya valores de consanguinidad (Tabla 1).

Asimismo, en Canadá se realizó una evaluación de la consanguinidad, observándose que los valores hallados son mayores que los norteamericanos (Doormaal, 2008), que se puede observar en la Tabla 2.

Thompson et al. (2000), en un estudio, muestra el efecto que tiene la consanguinidad en la producción de leche, grasa y proteína, cuyos resultados se resumen en la Tabla 3, observándose que a medida que se incrementa la consanguinidad la depresión endogámica es más evidente.

Wiggans et al. (1995) realizaron un estudio en el cual cuantificaron el efecto del incremento de la consanguinidad en $1 \%$ sobre la producción de leche en algunas razas lecheras, los resultados se pueden ver en la Tabla 4.

Según Cassell (1999), en el ganado lechero se pueden presentar tres apareamientos específicos que los productores debieran tratar de evitar; el tipo de cruzamiento y sus efectos pueden ser observados en la Tabla 5.

Se han efectuado comparaciones con respecto al índice de fertilidad y mortalidad embrionaria en vacas consanguíneas inseminadas con semen procedente de toros con y sin parentesco. En el primer caso, cuando la madre y el feto son consanguíneos, la mortalidad embrionaria es más alta que cuando únicamente es consanguínea la madre. En comparaciones realizadas entre vacas consanguíneas y no consanguíneas se demostró que la mortalidad embrionaria era un $15 \%$ más elevada para las primeras que para las últimas. Cuando se unieron toros consanguíneos con vacas consanguíneas y emparentadas, el porcentaje de

Tabla 1. Valores promedios de consanguinidad en ganado vacuno lechero

\begin{tabular}{|l|c|c|}
\hline \multicolumn{1}{|c|}{ Raza } & Número de animales y parientes & $\begin{array}{c}\text { Nivel promedio de } \\
\text { consanguinidad (\%) }\end{array}$ \\
\hline Ayrshire & 20.605 & 4,7 \\
Guernsey & 104.951 & 3,5 \\
Holstein & 1.136 .123 & 2,6 \\
Jersey & 112.562 & 3,3 \\
Brown Swiss & 27.729 & 3,0 \\
Milking Shorthorn & 7.948 & 4,1 \\
\hline
\end{tabular}

Fuente: Wiggans, Van Raden y Zuurbier (1995). 
Tabla 2. Niveles de consanguinidad y su incremento en Canadá

\begin{tabular}{|l|c|c|c|c|c|}
\hline \multirow{2}{*}{\multicolumn{1}{c|}{ Raza }} & \multirow{2}{*}{$\begin{array}{c}\text { Promedio en \% para } \\
\text { consanguinidad al 2007 }\end{array}$} & \multicolumn{4}{c|}{$\begin{array}{c}\text { Aumento medio anual en promedio } \\
\text { Porcentaje de la endogamia }\end{array}$} \\
\cline { 3 - 6 } & & $1970-1979$ & $1980-1989$ & $1990-1999$ & $2000-2007$ \\
\hline Ayrshire & 5,94 & .23 & .19 & .08 & .04 \\
\hline Brown Swiss & 5,36 & .05 & .21 & .13 & .12 \\
\hline Canadiense & 7,93 & .08 & .29 & .20 & .23 \\
\hline Guernsey & 5,65 & .04 & .08 & .19 & .15 \\
\hline Holstein & 5,75 & .12 & .08 & .27 & .08 \\
\hline Jersey & 5,82 & .13 & .05 & .15 & .10 \\
\hline Milking Shorthorn & 2,13 & .04 & .00 & .27 & -.13 \\
\hline
\end{tabular}

Tabla 3. Niveles de consanguinidad y su efecto en la cantidad y calidad de la leche

\begin{tabular}{|c|c|c|c|}
\hline $\mathrm{F}_{\mathrm{x}}(\%)$ & Leche $(\mathrm{kg})$ & Grasa $(\mathrm{kg})$ & Proteína $(\mathrm{kg})$ \\
\hline 2,00 & $-36,24$ & $-1,56$ & $-0,22$ \\
4,00 & $-116,34$ & $-4,21$ & $-2,83$ \\
6,00 & $-180,68$ & $-7,13$ & $-4,46$ \\
8,00 & $-300,35$ & $-10,00$ & $-7,74$ \\
10,00 & $-395,53$ & $-13,83$ & $-10,81$ \\
16,90 & $-630,30$ & $-21,54$ & $-17,81$ \\
26,40 & $-707,78$ & $-23,33$ & $-19,93$ \\
\hline
\end{tabular}

Tabla 4. Efecto de la consanguinidad en la producción de leche

\begin{tabular}{|c|c|}
\hline Raza & Efecto del incremento del 1\% de consanguinidad \\
\hline Holstein & $-29,48 \mathrm{~kg}$ \\
Ayrshire & $-30,39 \mathrm{~kg}$ \\
Guernsey & $-19,50 \mathrm{~kg}$ \\
Jersey & $-21,32 \mathrm{~kg}$ \\
Brown Swiss & $-24,49 \mathrm{~kg}$ \\
\hline
\end{tabular}

Tabla 5. Tipo de apareamiento y su efecto en la producción de leche

\begin{tabular}{|c|c|c|c|}
\hline \multirow{2}{*}{$\begin{array}{c}\text { Cruzamiento del toro } \\
\text { con: }\end{array}$} & $\begin{array}{c}\text { Porcentaje de } \\
\text { Endogamia }\end{array}$ & $\begin{array}{c}\text { Cambios promedio esperados } \\
\text { primera lactación }(\mathrm{kg})\end{array}$ & $\begin{array}{c}\text { Proteína durante la } \\
\text { primera lactación }(\mathrm{kg})\end{array}$ \\
\hline Su propia hija & $25,00 \%$ & 929,86 & 34,01 \\
\hline $\begin{array}{c}\text { Su propia media } \\
\text { hermana }\end{array}$ & $12,50 \%$ & 464,93 & 17,23 \\
\hline $\begin{array}{c}\text { La hija de su medio } \\
\text { hermano }\end{array}$ & $6,25 \%$ & 232,69 & 8,62 \\
\hline
\end{tabular}


gestaciones diagnosticadas fue solamente de un $36,8 \%$ comparado con el $65,7 \%$ para los apareamientos entre animales no emparentados y no consanguíneos. Es por tanto evidente que la influencia de la consanguinidad sobre la gestación es considerable (Johansson y Rendel, 1972). Smith (1997) reportó un aumento en el intervalo entre partos de 0,26 días por punto porcentual de aumento en consanguinidad, aumento de 0,36 días de edad al primer parto por $1 \%$ de consanguinidad, fertilidad más deficiente e incidencia más alta de muerte embrionaria temprana. Thompson et al. (2000) citado por Caraviello (2004), encontraron que la edad al primer parto aumentó hasta 26 días y la duración de la lactancia se redujo entre 2 y 8 días, cuando los niveles de consanguinidad fueron superiores al $10 \%$.

Información y registros de producción

Los registros de producción y la genealogía pertenecen a una empresa ganadera dedicada a la producción de leche, situada en el distrito de Lurín. La información de la genealogía se ha tomado de los registros propios de la empresa, así como de los certificados de genealogía expedidos por la Asociación de Criadores de Ganado Brown Swiss, así como de los registros del USDA (2010). Asimismo, se ha tomado la información de producción y reproducción de los 136 animales que se encuentran en la explotación y, a partir de ello, se ha completado la genealogía por la vía paterna y materna, encontrándose 820 animales emparentados genealógicamente en 15 generaciones.

Métodos para determinar los coeficientes de consanguinidad

Los métodos clásicos para determinar el coeficiente de consanguinidad, son los descritos por Wright (1922) y Malecot (1969). La ecuación para determinar el coeficiente de consanguinidad es la siguiente:

$$
\mathrm{F}_{x=1 / 2 \sum\left[(1 / 2)^{\mathrm{n}}(1+\mathrm{F} a)\right]}
$$

$F_{x} F_{x}$ : Coeficiente de consanguinidad del individuo $\mathrm{X}$. $\Sigma$ : Sumatoria.

$n n$ : Numero de vías que conecta a los padres del macho a través de un antecesor en común.

$F_{a} F_{a}$ : Coeficiente de consanguinidad del antecesor común en cada camino.

\section{Cálculo de los coeficientes de consanguinidad.}

Se utilizó el programa ENDOG (Gutiérrez y Goyache, 2005), que es un software que nos permite calcular los coeficientes de consanguinidad, así como la matriz de las relaciones aditivas, adicionalmente permite determinar ciertos valores poblacionales, como el tamaño efectivo de la población, intervalos entre generaciones y distribución de los animales de población en las generaciones y ancestros.

\section{De las características a evaluar}

Se evaluaron las características reproductivas de las vaquillonas en la edad del primer parto y el número de servicios efectivos para la preñez. El modelo matemático para analizar el número de servicios efectivos para la preñez del primer parto fue:
$N^{\circ}$ Servicios $=\mu+$ Año de Parto + Edad al Parto $+F_{x}+\varepsilon_{i j}$ La edad al parto fue considerada como una covariable para ajustar los datos a la edad, ya que es un factor que influye en las características productivas y reproductivas, $F_{x}$ representa al grupo de consanguinidad al que pertenece el animal.

En cuanto a las características en vacas, se ha considerado el número de servicios efectivos para la preñez, número de partos (longevidad), duración de la lactación promedio (DIM), intervalo entre partos y producción de leche. El modelo matemático para el intervalo entre partos y para la producción de leche fue el siguiente:

$I P /$ Prod.Leche $=\mu+N^{\circ}$ Parto + Año Parto + Edad al Parto $+F_{x}+\varepsilon_{i j}$ donde:

IP = Intervalo entre parto, producción de leche, la edad al parto fue considerada como una covariable, $F_{x}$ es el grupo de consanguinidad a la que pertenece el animal. Los datos fueron analizados utilizando el paquete estadístico SAS (2001), con el procedimiento Proc GLM.

\section{Resultados y discusión}

\section{Estimación y evaluación de la consanguinidad}

Los valores de la consanguinidad han sido estimados utilizando el programa ENDOG (Gutiérrez y Goyache, 2005); de los 136 animales evaluados, completada la genealogía, se ha obtenido 820 animales emparentados, formándose 15 generaciones. Los valores de los coeficientes de consanguinidad, a través de las 15 generaciones, se muestran en la Tabla 6. Como podrá observarse, a partir de la cuarta generación comienza a notarse ya la consanguinidad en el grupo genealógico, correspondiendo al $12,50 \%$ de los animales y siendo más constante la presencia de animales consanguíneos a partir la sexta generación; además, en las generaciones 14 y 15 todos los animales son consanguíneos, lo que significa que los reproductores machos, principalmente, utilizados mediante inseminación artificial, tienen una relación de parentesco que incrementa la posibilidad de formar individuos consanguíneos.

De los 136 animales del rebaño, determinado sus coeficientes de consanguinidad, se han formado los cinco grupos en función al valor de la consanguinidad; dicha distribución se presenta en la Tabla 7.

Efecto de la consanguinidad sobre los caracteres reproductivos al 1 er parto

Edad al primer parto y número de servicios efectivo para la preñez

Analizando los grupos formados, en cuanto a la edad del primer parto, los grupos consanguíneos presentaron en promedio mayor edad en el parto que la de los animales no consanguíneos, indudablemente el hecho hace suponer que la consanguinidad, en promedio, afecta en tener 20 días más de edad para el primer parto (ver Tabla 8). Esto afecta los ingresos de la empresa ya que inician la lactación a una mayor edad.

Analizando el número de servicios de acuerdo a la preñez de las vaquillas, se ha observado que en promedio los grupos consanguíneos necesitan de un número mayor de servicios para la preñez que los grupos no consanguíneos. Dichos resultados concuerdan con lo establecido por 
Tabla 6. Coeficientes de consanguinidad y población consanguínea

\begin{tabular}{|c|c|c|c|c|}
\hline Generación & $\mathrm{N}$ & $\begin{array}{c}\text { Consanguinidad en la } \\
\text { población, } \%\end{array}$ & $\begin{array}{l}\text { \% de la Población } \\
\text { consanguínea }\end{array}$ & $\begin{array}{c}\text { Consanguinidad } \\
\text { Promedio, } \%\end{array}$ \\
\hline 0 & 110 &.-- &.-- & -.- \\
\hline 1 & 24 &.-- &.-- &.-- \\
\hline 2 & 14 &.-- &.-- &.-- \\
\hline 3 & 13 &.-- &.-- &.-- \\
\hline 4 & 16 &.-- & 12,50 & 6,25 \\
\hline 5 & 30 &.-- &.-- &.-- \\
\hline 6 & 55 & 0,05 & 10,91 & 0,46 \\
\hline 7 & 63 & 0,81 & 41,27 & 1,97 \\
\hline 8 & 99 & 1,03 & 53,54 & 1,92 \\
\hline 9 & 116 & 1,50 & 81,90 & 1,83 \\
\hline 10 & 106 & 1,72 & 81,13 & 2,12 \\
\hline 11 & 91 & 2,25 & 92,31 & 2,44 \\
\hline 12 & 54 & 2,34 & 87,04 & 2,69 \\
\hline 13 & 23 & 2,62 & 78,26 & 3,35 \\
\hline 14 & 5 & 0,91 & 100,00 & 0,91 \\
\hline 15 & 1 & 1,23 & 100,00 & 1,23 \\
\hline
\end{tabular}

Tabla 7. Distribución de las vacas en función al valor de la consanguinidad

\begin{tabular}{cccc}
\hline Grupo & $\begin{array}{c}\text { Intervalo de } \\
\text { Consanguinidad }\end{array}$ & Número de animales & $\begin{array}{c}\text { Valor promedio de } \\
\text { consanguinidad }\end{array}$ \\
\hline 1 & $0 \%$ & 21 & $0,00 \%$ \\
2 & $(0 \%-2 \%]$ & 62 & $0,94 \%$ \\
3 & $(2 \%-4 \%]$ & 29 & $2,63 \%$ \\
4 & $(4 \%-6 \%]$ & 16 & $4,82 \%$ \\
5 & $(6 \%$ a más] & 8 & $8,74 \%$ \\
\hline
\end{tabular}

Tabla 8. Valores promedios de edad al primer parto y número de servicios a la preñez en vaquillas

\begin{tabular}{ccccccc}
\hline \multirow{2}{*}{ Grupo } & \multicolumn{2}{c}{ Edad al primer parto (meses) } & & \multicolumn{3}{c}{ Número de servicios } \\
\cline { 2 - 3 } & $\mathrm{N}$ & promedio & sig & $\mathrm{n}$ & promedio & sig \\
\hline 1 & 21 & 25,7524 & $\mathrm{~b}$ & 21 & 1,8571 & $\mathrm{~b}$ \\
2 & 57 & 26,9684 & $\mathrm{a}$ & 62 & 2,2581 & $\mathrm{a}$ \\
3 & 27 & 25,9852 & $\mathrm{ab}$ & 29 & 1,9655 & $\mathrm{ab}$ \\
4 & 13 & 26,5231 & $\mathrm{a}$ & 16 & 1,8750 & $\mathrm{~b}$ \\
5 & 6 & 26,0667 & $\mathrm{a}$ & 8 & 2,1250 & $\mathrm{a}$ \\
\hline
\end{tabular}

Letras diferentes indican diferencias significativas $(\mathrm{P}<0,05)$.

Falconer y Makay (1966) y Cassell (1999), quienes indican que la consanguinidad afecta los valores reproductivos en las vacas lecheras.

Efecto de la consanguinidad sobre los caracteres reproductivos y productivos en vacas

Número de servicios promedio para la preñez efectiva y duración de la lactación (DIM)

$\mathrm{Al}$ analizar el número de servicios para la preñez efectiva en vacas de más de un parto, se observó que la consanguinidad tuvo un efecto negativo sobre este carácter, ya que el grupo no consanguíneo mostro el menor número de servicios para la preñez efectiva (2,08 servicios/preñez).

Cuando se analizó la permanencia de los animales dentro del rebaño, medido como número de parto, el grupo no consanguíneo es el que presentó un mayor número de partos durante su vida productiva, hecho que podemos relacionar con una mayor longevidad (3,12 lactaciones), mientras que, en promedio, los grupos consanguíneos mostraron menor número de partos o menor longevidad, a excepción del grupo 4 que mostró una media de 3,17 lactaciones, similar al grupo no consanguíneo. 
Tabla 9. Valores promedio de los parámetros reproductivos en vacas lecheras

\begin{tabular}{|c|c|c|c|c|c|c|}
\hline \multirow{2}{*}{ Grupo } & \multicolumn{2}{|c|}{ Número de servicios } & \multicolumn{2}{|c|}{ Número de partos } & \multicolumn{2}{|c|}{ Días en lactación (DIM) } \\
\hline & $\mathrm{n}$ & promedio & $\mathrm{n}$ & Promedio \pm ES & $\mathrm{n}$ & Promedio \pm ES \\
\hline 1 & 100 & $2,08 \mathrm{ab}$ & 100 & $3,12 \pm 0,18$ & 89 & $257,96 \pm 13,21$ \\
\hline 2 & 138 & $2,70 \mathrm{a}$ & 138 & $2,32 \pm 0,14$ & 106 & $250,81 \pm 12,97$ \\
\hline 3 & 52 & 2,92 a & 52 & $1,90 \pm 0,16$ & 34 & $307,47 \pm 32,00$ \\
\hline 4 & 35 & $2,20 \mathrm{ab}$ & 35 & $3,17 \pm 0,44$ & 26 & $261,85 \pm 25,54$ \\
\hline 5 & 8 & $1,75 \mathrm{~b}$ & 8 & $1,37 \pm 0,26$ & 6 & $199,00 \pm 46,60$ \\
\hline
\end{tabular}

Letras diferentes indican diferencias significativas $(\mathrm{P}<0,05)$.

Tabla 10. Intervalos entre partos promedio en vacas lecheras

\begin{tabular}{ccccc}
\hline Grupo & $\mathrm{N}$ & Promedio & $\begin{array}{c}\text { Error } \\
\text { Estándar }\end{array}$ & significancia \\
\hline 1 & 73 & 439,40 & $\pm 8,27$ & $\mathrm{~b}$ \\
2 & 64 & 430,83 & $\pm 13,58$ & $\mathrm{~b}$ \\
3 & 17 & 539,76 & $\pm 34,46$ & $\mathrm{a}$ \\
4 & 19 & 426,11 & $\pm 19,12$ & $\mathrm{~b}$ \\
5 & 2 & 476,00 & $\pm 115,0$ & $\mathrm{~b}$ \\
\hline
\end{tabular}

Con respecto a la duración de la lactación (DIM), no se ha mostrado un efecto considerable, ya que los grupos de animales han promediado valores de DIM muy variados, lo cual indicaría que no hay un efecto marcado de la consanguinidad en la duración de las lactaciones; el grupo no consanguíneo promedió una duración de la lactación de 257,95 días, mientras que en los grupos consanguíneos los valores medios fueron similares. Dichos valores, presentan una relación con lo encontrado en la literatura, como Cassell (1999), Thompson et al. (2000), Wiggans et al. (1995). Las diferencias indicadas se muestran en la Tabla 9 , donde se podrá observar los valores para los grupos de animales en el presente estudio.

\section{Intervalo entre partos}

Al analizar el intervalo entre partos presentados en los grupos de los animales, se ha observado que los grupos consanguíneos medios y altos presentaron mayores valores de intervalo, lo cual afecta la producción vitalicia, ya que al tener intervalos prolongados hay una mayor cantidad de días vacíos, un mayor número de servicios, mientras que el grupo no consanguíneo presentó un valor bajo $(439,40$ días). Los valores promedio encontrados en los grupos de consanguinidad se pueden observar en la Tabla 10.

En cuanto a la producción de leche, a pesar de que el análisis de varianza mostró un efecto altamente significativo en los niveles de consanguinidad, al realizarse el análisis de medias, estas no presentaron diferencias estadísticas, a excepción del grupo de alta consanguinidad $\left(F_{x}>6,00 \%\right)$. Si bien es cierto que no hay diferencias estadísticas, sí hay diferencias numéricas, no encontrándose ninguna relación ya que los grupos consanguíneos presentaron un mejor promedio de producción de leche, lo que no coincide con lo establecido en la literatura, que indican que la depresión endogámica se muestra en la característica de producción de leche, observándose una reducción en sus valores (Cassell, 1999; Wiggans et al., 1992).

\section{Conclusiones}

La consanguinidad, en vaquillonas, incrementa la edad del primer parto y el número de servicios para la preñez efectiva. En vacas, la consanguinidad influye en los parámetros reproductivos de la misma manera que en el caso de las vaquillonas ya que se requiere un número mayor de servicios para la preñez efectiva debido a la longevidad de las vacas y a un mayor intervalo entre partos, lo que indirectamente señala sus efectos sobre el aspecto reproductivo.

En cuanto al efecto de la consanguinidad sobre la producción de leche, no se observa un efecto negativo, mostrando valores altos de producción de leche en los grupos consanguíneos en comparación con los animales que no lo son.

\section{Literatura citada}

Caraviello, D. 2004. Selección Genética. Guía Técnica Lechera - Suplemento. The Babcock Institute for International Dairy Research and Development. University of Wisconsin, Madison, Wisconsin, USA. Recuperado de http://www.itgganadero.com

Cassell, B. 1999. Inbreeding. Extension Dairy Scientist. Publicación No 404-080. Recuperado de http://www.ext. vt.edu/pubs/dairy/404-080/404-080.html

Doormaal, B. 2008. Inbreeding Update. Recuperado de http://www.cdn.ca/Articles/inbreeding_update.htm.

Falconer, D.S. y Mackay, T.F. 1996. Introducción a la genética cuantitativa. (4ta. ed.) Nueva York - Estados Unidos: John Wiley \& Sons, Inc.

Gutiérrez, J. P. y Goyache, F. 2005. A note on ENDOG: a computer program for analyzing pedigree information. Journal of Animal Breeding and Genetics, 122, 172-176.

Gutierrez, J.P.; Goyache, F. y Cervantes, I. 2009. User's Guide. Endog 4.6. A computer program for monitoring genetic variability of populations using pedigree information. 
Johansson, I. y Rendel, J. 1972. Genética y Mejora Animal. Zaragoza, España: Editorial Acribia.

Mujica, F. 1992. Hibridismo en producción animal. En Latrille, L. (ed). Producción animal. Valdivia, Chile: Universidad Austral de Chile. Facultad de Ciencias Agrarias, Instituto Producción Animal; Serie B - 16.

Neira, R. 1985. Introducción al estudio de la consanguinidad en animales. Serie publicación docente, $\mathrm{N}^{\mathrm{o}}$ 11. Santiago, Chile.

SAS. 2001. SAS/STAT User's Guide. (Release 8.20). Cary, NC, USA. SAS Inst. Inc.

Smith, L. A. 1997. The effect of inbreeding on lifetime performance of dairy cattle. (Tesis Master of Science). Department of Dairy Science, Virginia Polytechnic Institute, and State University, Blacksburg. J. Dairy Sci, 81: 2729-2737. Recuperado de http://jds.fass.org/cgi/ reprint/81/10/2729

Thompson, J.R.; Everett, R.W.y Hammerschmidt, N.L. 2000a. Effects of Inbreeding on Production and Survival in Holstein. J. Dairy Sci, 83, 1856-1864. Recuperado de http://jds.fass.org/cgi/reprint/83/8/1856

Thompson, J.R.; Everett, R.W. y Wolfet, C. $2000 \mathrm{~b}$. Effects of inbreeding on production and survival in Jersey. J. Dairy Sci, 83: 2131 - 2138. Recuperado de http://jds. fass.org/cgi/content/abstract/83/9/2131

Wiggans, G.R.; Van Raden, P.M. y Zuurbier, J. 1995. Cálculo y utilización de los Coeficientes de Consanguinidad para Evaluación Genética de Estados Unidos el ganado lechero. J. Dairy Sci, 78: 1584-1590. Recuperado de http:// jds.fass.org/cgi/reprint/78/7/1584.pdf

Wright, S. 1922. Coefficient of inbreeding and relationship. American Naturalist magazine, 56: 330338. United States. Recuperado de http://aipl.arsusda.gov/ publish/other/wright1922.pdf 